\title{
Cooperativity and resonances in periodically driven spin-boson systems
}

\author{
Feng Shuang, Chen Yang, Houyu Zhang, and YiJing Yan* \\ Department of Chemistry, Hong Kong University of Science and Technology, Kowloon, Hong Kong \\ and Open Laboratory of Bond-Selective Chemistry, University of Science and Technology of China, Hefei, China
}

(Received 24 November 1999)

\begin{abstract}
We present an analytical recursive formulation for the quantum transport in symmetric two-level systems under the influence of both dissipation and periodic driving. The rate-matching condition for quantum stochastic resonance despite its different appearance is found to be physically the same as that in the classical case. Analyzed are also the Rabi resonance and its implication to quantum stochastic resonance. We demonstrate that no matter how weak the driving field is, transport can involve about $70 \%$ population in the vicinities of the third-harmonic as well as the fundamental-harmonic Rabi resonance. Recovered is also an adiabatic passage condition in which the transport carries a nearly $100 \%$ population in the low frequency and strong driving limit.
\end{abstract}

PACS number(s): 05.30.- d, 05.40.-a, 42.65.Ky, 73.50.Td

Cooperativity plays an important role in nonlinear science. Stochastic resonance (SR) is a kind of cooperative effect in which the response of physical systems to periodic driving is maximized at a certain level of noise. Since its discovery in 1981 [1], SR has been recognized as one of general nonlinear phenomena occurring in a variety of fields of science (for recent reviews, see, e.g., [2] and the references therein). The canonical model for classical SR is described by a periodically driven bistable state system embedded in a fluctuating medium (or noise) [2]. The transport amplitude maximum is found at the noise level such that its induced transport rate matches with that induced by the periodic driving [2]. This is the synchronization principle or the rate-matching condition of SR. In the case of classical transport, it is given [2] by $r_{K}=\Omega / 2$. Here, $r_{K}$ is the Kramers rate for barrier crossing, while $\Omega$ is the driving frequency. The factor $1 / 2$ accounts for the periodically driven transport occurring in both forward and backward directions.

The role of SR in quantum transport systems, such as nonlinear optics and semiconductor devices, has been a subject of many recent studies [2-6]. The most important feature of quantum SR that differs from its classical counterpart appears in the vicinity of resonant driving region $[5,6]$. It has been shown theoretically [5] that no matter how weak the driving field is, the fundamental-harmonic transport amplitude that involves about $70 \%$ of population can occur at the resonant driving condition with appropriate noise levels. In this work we shall demonstrate that a large transport amplitude might be achievable not only in the fundamental but also the third-order resonance region. It thus implies the possibility of an efficient way to frequency tripling by using a weak driving field. This paper also provides an analytical expression to the study of quantum transport in a model dissipative two-level system (TLS) driven by arbitrary periodical fields at arbitrary temperatures.

The simplest model for quantum SR is a periodically driven spin-boson system, or a symmetric TLS under the

\footnotetext{
*Author to whom correspondence should be addressed. Electronic address: yyan@ust.hk
}

influence of both noise and periodic driving [2-6]. The total Hamiltonian assumes

$$
H_{T}(t)=-(\hbar / 2)\left\{c \hat{\sigma}_{x}+[b \cos \Omega t+f(t)] \hat{\sigma}_{z}\right\} .
$$

Here, $\hat{\sigma}_{x}$ and $\hat{\sigma}_{z}$ are the Pauli spin operators. $c$ denotes the coupling matrix element of the bare TLS, $b$ the strength of periodic driving, and $\Omega$ the driving frequency. In Eq. (1), $f(t)$ represents the quantum Markovian random force whose correlation function is characterized by the noise-level parameter $\gamma$. In this case, Eq. (1) leads to the following Bloch equations:

$$
\begin{gathered}
\dot{S}_{x}(t)=-\gamma\left[S_{x}(t)-\bar{S}_{x}(t)\right]+b \cos (\Omega t) S_{y}(t), \\
\dot{S}_{y}(t)=-b \cos (\Omega t) S_{x}(t)-\gamma S_{y}(t)+c S_{z}(t), \\
\dot{S}_{z}(t)=-c S_{y}(t) .
\end{gathered}
$$

Here, $S_{r}(t) \equiv \operatorname{Tr}\left[\hat{\sigma}_{r} \rho(t)\right]$; with $r=x, y, z$ are the expectation values of three Pauli spin-operators, and

$$
\begin{gathered}
\bar{S}_{x}(t)=\left(c / \omega_{t}\right) \tanh \left[\hbar \omega_{t} /\left(2 k_{B} T\right)\right], \\
\omega_{t} \equiv \sqrt{c^{2}+b^{2} \cos ^{2}(\Omega t)} .
\end{gathered}
$$

Note that $\omega_{t}$ is the instantaneous transition frequency of the periodically driven TLS, while $\bar{S}_{x}(t)=\operatorname{Tr}\left[\sigma_{x} \bar{\rho}(t)\right]$ with $\bar{\rho}(t)$ denoting the instantaneous Boltzmann density matrix at temperature $T$. As $\bar{S}_{y}=0$ and $\bar{S}_{z}=b \cos (\Omega t) \bar{S}_{x} / c$, the Bloch equations [Eq. (2)] do not contain them explicitly. In the high temperature limit of $k_{B} T \gg \hbar \omega_{t}$, we have $\bar{S}_{x}(t) \approx c /\left(2 k_{B} T\right)$ $\ll 1$. In this case Eq. (2) reduces to the Bloch equations used by Pareek et al. [6] in their study of quantum SR in the weak driving $(b \ll c)$ regime. However, it is crucial especially in the low temperature $\left(k_{B} T<\hbar \omega_{t}\right)$ and strong driving $(b>c)$ regime to have the instantaneous field-dressed Boltzmann value of $\bar{S}_{x}(t)$ in Eq. (2). If it were replaced by the field-free value of $S_{x}^{\text {eq }}$, Eq. (2) would lead to the unphysical result of negative population. 
In this work, we shall be interested in the stationary solution to Eq. (2), which according to the Floquet theorem is of the form: $S_{r}^{(s)}(t) \equiv \frac{1}{2} \sum r_{n} e^{-i n \Omega t}$. Here, $n \in(-\infty, \infty)$, and $r_{-n}=r_{n}^{*}$, with $r_{n}=x_{n}, y_{n}$, or $z_{n}$. The stationary dynamics of Eq. (2) can then be studied in terms of $\left\{r_{n}\right\}$ via their coupled linear equations. To that end, we shall also decompose Eq. (3a) as $\bar{S}_{x}(t) \equiv \sum s_{n} e^{-i n \Omega t}$. Obviously, $s_{2 m+1}=0$, and $s_{n}=s_{-n}=s_{n}^{*}$. These real parameters constitute the constant vector $[\mathrm{cf}$. Eq. (4c)] in the linear equation for the stationary harmonic amplitudes $\left\{r_{n} ; n \geqslant 0\right\}$. The symmetry of the TLS [Eq. (1)] leads to $x_{2 m+1}=y_{2 m}=z_{2 m}=0$, and the remaining linear equation involves effectively only a symmetric tridiagonal matrix. The stationary harmonic amplitudes can thus be formulated analytically. To simplify the notation, we denote $\alpha_{m}$ and $\beta_{m}$ as the diagonal and the offdiagonal elements of the symmetric tridiagonal matrix, and $\left\{v_{m}\right\}$ as the constant vector. We have

$$
\begin{gathered}
\alpha_{m} \equiv \frac{c^{2}}{i(2 m+1) \Omega}+i(2 m+1) \Omega-\gamma+\beta_{m}+\beta_{m-1}, \\
\beta_{m} \equiv \frac{b^{2} /(4 \gamma)}{i(2 m+2) \Omega / \gamma-1}, \\
v_{m}=b\left[\frac{s_{2 m+2}}{1-i(2 m+2) \Omega / \gamma}+\frac{s_{2 m}}{1-i(2 m) \Omega / \gamma}\right]
\end{gathered}
$$

Let us present the final analytical formulation for the stationary harmonic transport amplitudes $z_{2 m+1}$, or

$$
S_{z}^{(s)} \equiv\left[\rho_{11}(t)-\rho_{22}(t)\right]_{s}=\operatorname{Re} \sum_{m=0}^{M} z_{2 m+1} e^{-i(2 m+1) \Omega t} .
$$

Here, $M$ is a sufficiently large truncation number such that Eq. (5) converges. We shall also set $\beta_{M} \equiv 0$. The final analytical solution is expressed as an inward-outward-recursion formulation as follows. We first construct inward-recursively $(m=M, \ldots, 0)$ two sets of auxiliary parameters:

$$
\begin{gathered}
\Gamma_{m}=\alpha_{m}-\beta_{m}^{2} / \Gamma_{m+1}, \\
\Lambda_{m}=\left(v_{m}-\beta_{m} \Lambda_{m+1}\right) / \Gamma_{m} .
\end{gathered}
$$

The stationary harmonic transport amplitudes are then evaluated outward recursively as

$$
\begin{aligned}
& z_{1}=\frac{\Gamma_{0}^{*}\left(v_{0}-\beta_{0} \Lambda_{1}\right)+\left[b^{2} /(4 \gamma)\right]\left(v_{0}-\beta_{0} \Lambda_{1}\right) *}{(i \Omega / c)\left\{\left|\Gamma_{0}\right|^{2}-\left[b^{2} /(4 \gamma)\right]^{2}\right\}}, \\
& z_{2 m+1}=\frac{\Lambda_{m}}{i(2 m+1) \Omega / c}-\frac{(2 m-1) \beta_{m-1}}{(2 m+1) \Gamma_{m}} z_{2 m-1} .
\end{aligned}
$$

Equations (4) - (7) constitute the exact stationary solution to Eq. (2) in the full $(c, b, \Omega, \gamma, T)$ parameter space. In the following we shall use them in a converged manner to investigate the transport dynamics at $T \rightarrow 0 \mathrm{~K}$ (or more precisely $\left.k_{B} T \ll \hbar c\right)$ in both the weak and the strong driving regimes, exemplified by $b / c=0.1$ and $b / c=5$, respectively. As the noise is longitudinal [cf. Eq. (1)], its effect will be reported

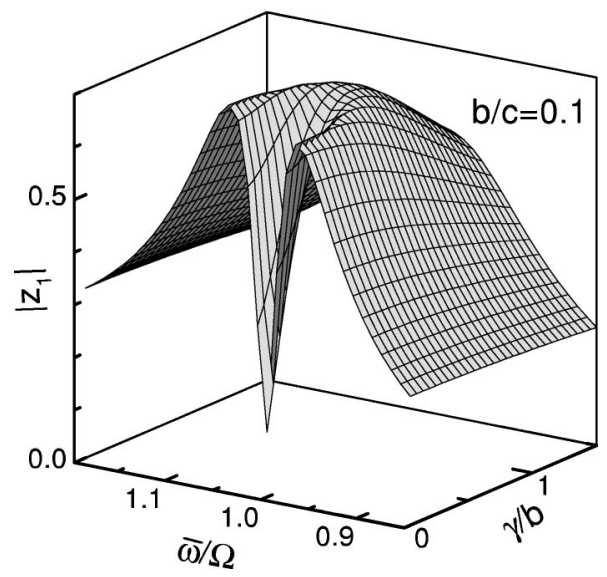

FIG. 1. The first-harmonic transport amplitude $\left|z_{1}\right|$ as a function of the noise level (in terms of $\gamma / b$ ) in the vicinity of first harmonic Rabi-resonance $\Omega \approx \bar{\omega}$. The specified value of $b / c=0.1$ is chosen to represent the weak driving case.

in term of $\gamma / b$. The effect of driving frequency $\Omega$ will be reported in the unit of the cyclic mean-square-root of the dressed-TLS frequency [Eq. (3b)],

$$
\bar{\omega} \equiv\left\langle\omega_{t}^{2}\right\rangle^{1 / 2}=\sqrt{c^{2}+b^{2} / 2} .
$$

We shall hereafter term $\bar{\omega}$ in Eq. (8) as the effective Rabi frequency of the driven TLS. It will be shown later that the quantum SR appears dramatically in the vicinity of the $(2 m+1)$ th-harmonic Rabi-resonance frequency $\Omega=\bar{\omega} /(2 m$ $+1)$, in both the weak and the strong driving regimes.

Let us start with the stationary quantum transport and its $\mathrm{SR}$ at the representative value of $b / c=0.1$ for the weak driving regime. Figure 1 depicts the first-harmonic amplitude $\left|z_{1}\right|$ as a function of $\gamma / b$ and $\bar{\omega} / \Omega$ in the vicinity of fundamental Rabi-resonant driving. Observed in this figure for the weak driving regime at $T \rightarrow 0 \mathrm{~K}$ are three important features as follows.

(i) SR in $\left|z_{1}\right|$ occurs only in the vicinity of the fundamental Rabi-resonance. We may denote the two frequencies associating with the maxima of $\left|z_{1}\right|_{\gamma \rightarrow 0}$ as $\Omega_{+}$and $\Omega_{-}$, respectively. Obviously, $\Omega_{-}<\bar{\omega}<\Omega_{+}$, and the SR occurs only when $\Omega \in\left(\Omega_{-}, \Omega_{+}\right)$[cf. Eq. (12)].

(ii) $\left|z_{1}\right|_{\Omega \approx \bar{\omega}}$ exhibits a dramatic SR effect. It starts from almost zero when $\gamma \rightarrow 0$ reaches at its maximum $\left|z_{1}\right|_{\max }$ $\approx 0.7$ when $\gamma=b / \sqrt{2}$, and then falls off as $\gamma$ increases further. The observed rate-matching condition for the quantum $\mathrm{SR}$ at the resonant driving frequency is therefore given by

$$
\gamma_{\mathrm{SR}}=b / \sqrt{2} \text {. }
$$

Note that $\gamma$ amounts to the noise-induced transport rate in the symmetric TLS in the absence of driving field. On the other hand, the cyclic-averaged rate induced by the periodic driving field is given by $b / \sqrt{2}$. Thus, Eq. (9) for the quantum $\mathrm{SR}$ is consistent with the synchronization principle established for classical SR [2] despite the different appearances in their rate-matching conditions.

(iii) $\left|z_{1}\right|_{\max } \approx 0.7$ which is independent of $b$ in the weak driving $(b \ll c)$ regime and appears on a parabolic curve in the $(\Omega, \gamma)$ space [cf. Fig. 1 and Eq. (11b)]. In this case, about 
$70 \%$ of population involves in the tunneling transport, no matter how weak the driving field is.

To analyze the above three important features of quantum transport in the weak driving regime at $T \rightarrow 0 \mathrm{~K}$, let us consider the lowest order truncation approximation $(M=0)$ to the exact formulation [Eqs. (6) and (7)]. After some elementary algebra, we obtain

$$
z_{1} \approx c F /\left(|F|^{2}+\Omega^{2} / 2\right),
$$

with $F=\left(c^{2}-\Omega^{2}+i \Omega \gamma\right) / b$. In deriving Eq. (10), we considered also the low temperature limit in which $\bar{S}_{x}(t) \approx 1$ [cf. Eq. (3a)]. At a finite temperature, Eq. (10) may include the scaling constant of $\left\langle\bar{S}_{x}(t)\right\rangle=s_{0}$. In the high temperature limit, this scaling constant is $s_{0} \approx c /\left(2 k_{B} T\right)$ and Eq. (10) recovers the result of Pareek et al. [6]. However, the high temperature $\left(k_{B} T \gg \hbar \omega_{t}\right)$ leads also to $\left|z_{1}\right| \ll 1$. The low temperature is required for a large amplitude transport. In the weak driving $(b \ll c)$ regime and in the vicinity of the fundamental resonant $\Omega \approx \bar{\omega}$ driving, Eq. (10) gives basically the identical numerical results as Fig. 1 obtained via the exact inward-outward-recursive formulation [Eqs. (6) and (7)].

We shall also be interested in the maximum value of $\left|z_{1}\right|$, which can be achieved when $|F|^{2}=\Omega^{2} / 2$ [cf. Eq. (10)]. We obtain for the weak driving $(b \ll c)$ regime that

$$
\begin{gathered}
\left|z_{1}\right|_{\text {max }} \approx 2^{-1 / 2} c / \Omega, \\
\gamma=\left[b^{2} / 2-\left(\Omega-c^{2} / \Omega\right)^{2}\right]^{1 / 2} .
\end{gathered}
$$

In the case of resonance driving $\Omega=c \approx \bar{\omega}$, Eq. (11a) becomes $\left|z_{1}\right|_{\text {max }} \approx 0.7$, while Eq. (11b) reduces to Eq. (9). We have thus recovered the features (ii) and (iii) of Fig. 1 as mentioned earlier. Equation (11b) can further be used to quantize the feature (i) of Fig. 1, i.e., the range of driving frequency $\Omega \in\left(\Omega_{-}, \Omega_{+}\right)$within which the SR might occur. In the weak driving regime, this range may be evaluated as the roots of Eq. (11b) in the limit of $\gamma \rightarrow 0$. We have

$$
\Omega_{ \pm} \approx \sqrt{c^{2}+b^{2} / 8} \pm b / \sqrt{8} .
$$

Note that $\left|z_{1}\right|_{\gamma \approx 0} \approx 0.7$ reaches its maximum at $\Omega=\Omega_{ \pm}$, while $\left|z_{1}\right|_{\gamma \approx 0} \approx 0$ at $\Omega \approx \bar{\omega}$.

We have thus analyzed the three important features in Fig. 1 that demonstrate clearly the cooperative effects of periodic driving and dissipation on the stationary transport in the TLS. The Rabi resonance is the cooperativity among the driving frequency $\Omega$, the Rabi frequency $\bar{\omega}$, and/or the driving strength $b$. In the vicinity of Rabi resonance, quantum SR occurs [Eq. (9)] as the cooperativity between the driving strength $b$ and the noise level $\gamma$. That $\left|z_{1}\right|_{\max } \approx 0.7$ being independent of the driving strength in the weak driving $(b$ $\ll c$ ) regime is definitely beyond the linear response theory. The validity of latter should also include the condition of $|F|^{2} \gg \Omega^{2} / 2$ [cf. Eq. (10)], which in the vicinity of resonant driving corresponds to $\gamma \gg b / \sqrt{2}$.

Figure 2 depicts the most striking cooperativity in the weak driving $(b \ll c)$ regime, which is in the vicinity of $\Omega$ $=\bar{\omega} / 3$ the third-order harmonic transport amplitude can also achieve at its maximum value of $\left|z_{3}\right|_{\text {max }} \approx 0.7$, no matter how weak the driving is. In this case, $\left|z_{3}\right|$ is the dominant contri-

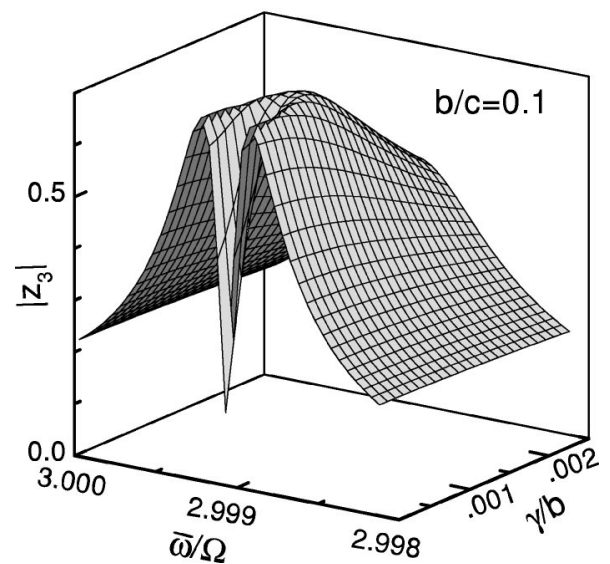

FIG. 2. Same as Fig. 1 but for $\left|z_{3}\right|$ in the vicinity of thirdharmonic Rabi resonance $\Omega \approx \bar{\omega} / 3$.

bution. Figure 2 for $\left|z_{3}\right|$ and Fig. 1 for $\left|z_{1}\right|$ are very much alike despite their difference measures in the $\Omega$ and $\gamma$ axes. The three characteristic features described earlier for Fig. 1 are also clearly seen in Fig. 2. Compared with the fundamental resonance in $\left|z_{1}\right|$ (Fig. 1), the third-harmonic Rabi resonance in $\left|z_{3}\right|$ (Fig. 2) occurs more sharply in both the driving-frequency $\Omega$ and the noise-level $\gamma$ domains.

Figure 3 plots both $\left|z_{3}\right|$ and $\left|z_{1}\right|$ as functions of driving frequency in the vicinity of third-harmonic Rabi resonance at the specified values of noise level and driving strength. Included is also the approximated $\left|z_{1}\right|$ (dash line) evaluated via the lowest-order truncation formulation [Eq. (10)]. Obviously, in the weak driving regime the third-harmonic transport amplitude $\left|z_{3}\right|$ whose maximum value is about 0.7 can be much larger than $\left|z_{1}\right|$ around $\Omega=\bar{\omega} / 3$. It thus leads to the failures of not only the linear response theory but also the lowest-order truncation approximation [Eq. (10)] at the thirdharmonic Rabi resonance, no matter how small the driving strength is.

We shall now turn to the strong $(b \gg c)$ driving regime exemplified by the chosen value of $b / c=5$. Figure 4 depicts the transport amplitudes, $\left|z_{1}\right|,\left|z_{3}\right|$, and $S_{z}^{\max }=\left|\rho_{11}-\rho_{22}\right|_{s}^{\max }$, as functions of the driving frequency at two specified noise levels. Note that $\left|z_{1}\right|$ can exceed 1 (cf. the upper panel of Fig. 4). However, $S_{z}^{\max } \leqslant 1$ (cf. the bottom panel) as it is required by the positivity of population. Evidently, $S_{z}^{\max } \rightarrow 1$ as

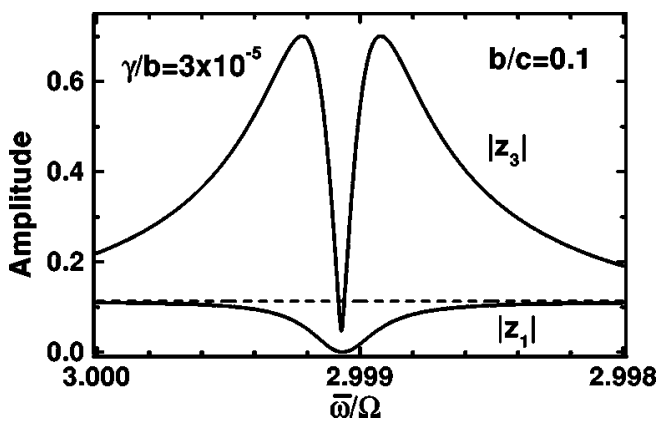

FIG. 3. $\left|z_{1}\right|$ and $\left|z_{3}\right|$ in the vicinity of third-harmonic Rabi resonance $\Omega \approx \bar{\omega} / 3$ in the weak driving regime, at the specified value of $\gamma$. The dotted line is the approximated $\left|z_{1}\right|$ via the lowest-order truncation formulation Eq. (10)]. 


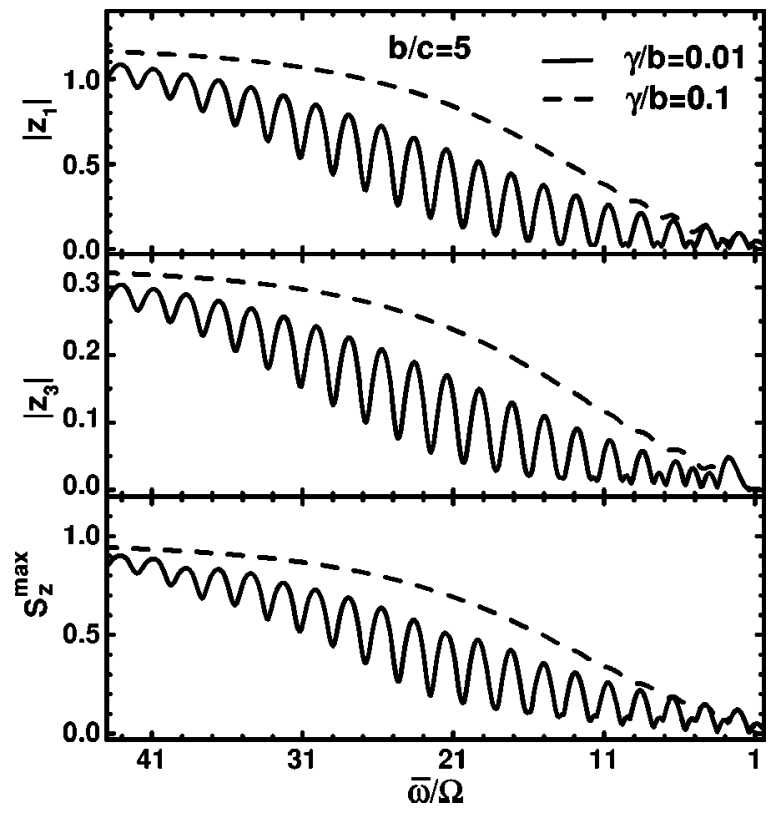

FIG. 4. The transport amplitudes as functions of $\bar{\omega} / \Omega$ at the specified value of $b / c=5$ to represent the strong driving regime. See text for the details.

$\Omega \rightarrow 0$. It implies that a $100 \%$ population transport occurs as a result of adiabatic passage in which $b \gg c \gg \Omega$. This phenomenon may be easily understood by realizing that in this case the region of Stark modulation, $(-b, b)$, exceeds that of tunneling transport, $(-c, c)$. Transport occurs only in a fraction of the driving period. By denoting $t_{T}$ as the duration of a forward or backward tunneling passage, we have $\sin \left(\Omega t_{T}\right)$ $=c / b$. For a given value of $b / c \gg 1$, the smaller the driving frequency $\Omega$, the longer the tunneling time $t_{T}$, and the more complete population transport. It is also interesting to notice that all three amplitudes in Fig. 4 behave similarly. This might result from the inward-outward recursive nature of the formulation, Eqs. (6) and (7), that correlates many harmonic amplitudes $z_{2 m+1}$ together in the strong driving regime. Within each of the Rabi-resonance regions (i.e., $2 m-1$ $<\bar{\omega} / \Omega<2 m+1)$, the transport amplitude in the strong driving regime behaves similarly as $\left|z_{1}\right|$ (Fig. 1) or $\left|z_{3}\right|$ (Fig. 2) in the vicinity of its own corresponding Rabi resonance in the weak driving regime. That is, the transport amplitude (e.g., $S_{z}^{\max }$ in the lower panel of Fig. 4) shows a frequency dip when $\gamma$ is sufficiently small (solid curves), and increases as $\gamma$ increases (dash curves) within a certain range. This indicates that SR occurs in each of the Rabi-resonance regions. Applying the synchronization principle, the rate-matching condition for the strong $(b / c \gg 1)$ driving regime would be $\gamma \sim t_{T}^{-1} \sim b \Omega / c$. However, the adiabatic passage would become the dominant mechanism at the low-frequency and strong driving regime, retaining a nearly $100 \%$ population transport as $\gamma$ increases further.

In summary we have reported a systematic study based on the analytical inward-outward recursive formulation [Eqs. (6) and (7)] for the quantum transport in a periodically driven spin-boson system. Elucidated are the stochastic resonance and Rabi resonance in both the weak and the strong driving regimes. Demonstrated are also the possibility of a large amplitude third-harmonic generation operated in the weak driving regime.

Support from the Research Grants Council of the Hong Kong Government and the National Natural Science Foundation of China is gratefully acknowledged. We would also like to thank Dr. Jixin Cheng for stimulating discussions and comments.
[1] R. Benzi, A. Sutera, and A. Vulpiani, Phys. Rev. A 14, L453 (1981); C. Nicolis and G. Nicolis, Tellus 33, 225 (1981).

[2] L. Gammaitoni, P. Hänggi, P. Jung, and F. Marchesoni, Rev. Mod. Phys. 70, 223 (1998).

[3] M. Grifoni and P. Hänggi, Phys. Rev. Lett. 76, 1611 (1996).

[4] R. Löfstedt and S. N. Coppersmith, Phys. Rev. Lett. 72,
1947 (1994).

[5] D. E. Makarov and N. Makri, Phys. Rev. B 52, R2257 (1995); Phys. Rev. E 52, 5863 (1995).

[6] T. P. Pareek, M. C. Mahato, and A. M. Jayannavar, Phys. Rev. B 55, 9318 (1997). 